\title{
THE METHODOLOGY OF TRAINING IN HEALTH CARE - A CONDITION AND FACTOR FOR SUCCESSFUL IMPLEMENTATION OF PATIENT SAFETY IN THE APPLICATION OF MEDICAL CARE
}

\author{
Elena Zheleva, Pepa Dzhedzheva \\ Department of Health Care, Sliven Affiliate, Medical University of Varna
}

ABSTRACT

INTRODUCTION: Higher education in school is a purposeful process of interaction between teachers and students. There are trained specialists suited to carry out professional activities and provide quality and safe health care for people. The methodology of training in health care offers safe, effective, individual, efficient, timely and equitable care.

AIM: The aim of the scientific report is to reveal and outline the organizational pedagogical conditions and the effectiveness of the methodology for the successful implementation of patient safety efforts in medical care. Patient safety is focused on the acquisition of knowledge, skills and competencies students need to work efficiently in the field of health care.

MATERIALS AND METHODS: The aim of the study is to reveal the role of methodology in the application of safe patient care; to investigate the effectiveness and its importance in patient safety; to establish the level of satisfaction with the application of safe patient care. The methods used during the research were: programmed interview with a survey and a pedagogical experiment. The subject of the research were students from the Nurse program, educators in health care, medical professionals, and patients. The object of the research were the process and the conditions under which the methodology was provided, a factor for successful implementation of patient safety when caring for patients.

RESULTS: The analysis of the results allowed to conclude that the teaching methodology in health care:

- is an important condition and factor in the successful implementation of patient safety (100\%);

- maintains, improves and enhances the acquisition of knowledge, skills and competencies of health professionals (98\%);

- is a key to addressing the needs of patients $(89 \%)$;

- satisfies the requirements of medical professionals to provide safe care for patients and their personal training (100\%).

\footnotetext{
Address for correspondence:

Elena Zheleva

Department of Health Care

Sliven Affiliate

Medical University of Varna

30 General Stoletov St

Sliven

e-mail:ejeleva@abv.bg
}

CONCLUSION: The methodology of training in health care is a condition and factor for success in providing safe and high quality care for patients.

Keywords: methodology of teaching health care, successful implementation, patient safety, training, future medical professionals, medical care 
Elena Zheleva, Pepa Dzhedzheva

\section{INTRODUCTION}

The education in higher education schools is a purposeful process of interaction between teachers and students, providing students, through modern methodology, with the needed skills for the organization and application of safe care. The applied patient safety measures lead to improved public health service. The methodology of training in health care creates the conditions for the application of quality and safe care for patients.

The safety of the patients part of the nursing care training of future specialists is associated with protection from unnecessary or potential harm. Preventable harm is a problem and a prerequisite for misuse of specific care. In the methodology of training in health care all medical procedures administered to patients play a role in their permanent protection from adverse events (2).

The methodology of training in health care provides methods and tools for successful implementation of patient safety in hospitals and outpatient settings. Trainees are provided with specialist knowledge about responsibility and acquire skills for the application of safe health care for everyone in need. The future specialists are trained to carry out professional activities without medical error accruing quality and safe health care for people (1).

Analyzing the content of the teaching methodology in health care found that it offers to the future professionals safe, effective, individual, efficient, timely and equitable care. Its main key objectives are: compliance, enforcement and ensuring the standards of general and special health care; proof to consumers of care that any medical care is up to date with modern medicine, which is instilled through the methodology to ensure patient safety; increasing the liability of future medical specialists caring for patients (4).

The methodology of the training of health care professionals is organized and conducted in school with specially selected topics. It aims at promoting the interests of students and their professional development. It retains all regulations, professional standards and ethics, professional development, training and covers the principles and norms of the profession of the medical specialist (1).
Modern advances in medicine worldwide are forcing future professionals to continuously seek new approaches to achieve their professional goals in a way that satisfies them offering a balance between professional and personal life. The most important goal of any professional is to achieve even better results while providing safe healthcare for patients, which is possible only through its continuous improvement and development. An important prerequisite in the teaching methodology in health care in an era of high technology is the adaptation of students to the latest approaches to resource management for safer healthcare for patients and their training (2).

The methodology of the training of health care professionals provides future medical professionals with the ability to: use techniques to identify the nature of type of care needed in order to cause no harm to the patient; formulate side effects of the type of care provided and how to prevent them; plan, measure and participate in the provision of safe medical care; communicate effectively with patients to ensure safety as an integral part of the care they receive; introduce strategies for patient safety; acquire knowledge about risk management. The techniques considered cover a wide range of areas of patient safety and aim to provide students with knowledge, skills and competencies related to patient safety in nursing care (1).

The methodology of training in health care is closely related to patient safety and quality of care. The development of scientific and technological innovations in medicine requires training in health care and developing competencies. Promoting patient safety leads to the application of quality care. The methods used are interactive. They include discussion of cases; formation of practical skills and competencies based on simulated patient role-playing games and using sophisticated dummies (3). They have many educational benefits and advantages related to patient safety: Students are taught, without jeopardizing the patient, to provide a safe working environment; all techniques can be repeated and adapted to the level and needs of future medical professionals; situations of emergency, in which students provide safe and quality care for the simulated patient are represented (4). 
The Methodology of Training in Health Care - a Condition and Factor for Successful Implementation ...

\section{AIM}

The aim of the scientific report is to reveal and outline the organizational pedagogical conditions and the effectiveness of the methodology for the successful implementation of patient safety efforts in medical care. Patient safety is focused on the acquisition of knowledge, skills and competencies by future medical professionals when providing health care.

The methodology of training health care professionals includes techniques of care provided for the patient and protection from complications.

\section{MATERIALS AND METHODS}

The objectives of the study are: to reveal the role of the teaching methodology in health care in ensuring safe care for patients; to investigate the effectiveness and its importance in patient safety; to establish the level of satisfaction from application of safe patient care. The used methods during the study are: programmed interview with a survey and a pedagogical experiment. The subject of the study are students from the Nurse program, educators in health care, medical professionals, and patients. The object of the study is the process and the conditions under which the methodology is provided - a factor for successful implementation of patient safety in caring for them.

\section{RESULTS AND DISCUSSION}

The methodology of training health care professionals presents information and knowledge about the optimal hospital environment; safe hygiene care; proper diet; optimal study of the vital organs and systems of the human body; communication with the patient and his relatives; rights and obligations of the patient; morality and ethics of the medical profession; safe use of medicines and physiotherapy.

The professional activities of future medical professionals are essential for the safety of patients in nursing care.

Analysis of the results allows to conclude that the teaching methodology in health care is a condition and factor in the successful implementation of patient safety (100\%). It systematically maintains, improves and enhances the acquisition of knowledge, skills and competencies of health care professionals $(98 \%)$. It is a key to addressing the needs of patients (89\%) and satisfies the requirements for medi- cal professionals to provide safe care for patients and the requirements of their personal professional training (100\%).

This study shows that the physical and mental fatigue of specialists, and the stressful conditions during their work shift are able to influence the safety of medical care. Of importance for the respondents is the patient, the type of care for him, teamwork, complexity of care, availability of necessary tools, organization of work, medical standards for the provision of specialized care, organization and management of health care, and the participation of the patient in the process of car, which minimizes the occurrence of errors.

The pedagogical experiment proved that the systematic approach to patient safety and the increase of the safety culture leads to a $99 \%$ reduction in the causes of critical incidents and injuries.

Teamwork and communication with other health professionals help keep track of errors in critical cases and use proper measures for patient safety in $92 \%$ of the cases.

All respondents are of the opinion that by the methods of training health care professionals patients' knowledge regarding their safety is improved.

The conducted programmed interview aimed to identify the needs of health professionals, which change with time according to the emerging needs of the patients when ensuring their safety in nursing care. The efficient use of resources in order to develop educational content when teaching methodology in health care allows the successful implementation of patient safety with their participation.

\section{CONCLUSION}

The methodology of training in health care is a factor for success in providing safe and quality care for patients. A training program related to safe medical care of patients was developed and presented. The patient safety culture should be part of the training of medical professionals and patients. A culture of safety behavior on the part of the respondents is required.

The successful implementation of patient safety requires the training of future medical professionals to be introduced and implemented in the curricula teaching methodology in health care. Health care 
professionals need to engage in continuing education at all levels of patient safety. During the safety training patients have to be included to present their point of view concerning the provision of safe medical care.

\section{REFERENCES}

1. Zheleva E. Methodology of practical training of medical professionals. EXPRESS; 2007.

2. Kircheva A. Safe Medicine. Stenograph; 2014.

3. WHO, London, 2006, Declaration of PFPS

4. http://ec.europa.eu/health/patient_safety/policy/ index_bg.htm. 